\title{
Counting statistics of dark-state transport through a carbon nanotube quantum dot
}

\author{
Nathan Ho $\odot$ and Clive Emary $\odot$ \\ Joint Quantum Centre Durham-Newcastle, School of Mathematics, Statistics and Physics, Newcastle University, \\ Newcastle upon Tyne NE1 7RU, United Kingdom
}

(Received 6 August 2019; revised manuscript received 16 October 2019; published 16 December 2019)

\begin{abstract}
In a recent experiment [A. Donarini et al., Nat. Commun. 10, 381 (2019)], electronic transport through a carbon nanotube quantum dot was observed to be suppressed by the formation of a quantum-coherent "dark state." In this paper we consider theoretically the counting statistics and waiting-time distribution of this dark-state-limited transport. We show that the statistics are characterized by giant super-Poissonian Fano factors and long-tailed waiting-time distributions, both of which are signatures of the bistability and extreme electron bunching caused by the dark state.
\end{abstract}

DOI: 10.1103/PhysRevB.100.245414

\section{INTRODUCTION}

In quantum optics, coherent population trapping is a phenomenon in which coherent illumination drives an electron into a particular superposition of orbital states - a dark statethat is decoupled from the light fields [1-3]. In Ref. [4], it was suggested that an all-electronic analog of this effect should exist in the transport through nanoelectronic systems such as a triple quantum dot. In this scenario, it is coherent tunneling between electronic states that permits the formation of a trapped state, and the "darkness" of this state is manifested as the suppression of electronic current through the system. Recently, Donarini et al. [5] reported the observation of current suppression in the transport through a carbon nanotube quantum dot (CNT-QD) and explained this effect as arising through the presence of a dark state formed by the superposition of longitudinal-orbital-momentum states in the nanotube.

In this paper we report on calculations of the counting statistics [6-12] and waiting-time distribution [13] of the nanotube model introduced in Ref. [5]. The counting statistics is a well established tool for obtaining information about transport processes beyond that which is available from measurements of the mean current alone [14-16]. Here we use the counting statistics formalism to investigate the current noise and skewness in particular. The waiting-time distribution, i.e., the distribution of times between consecutive electrontunneling events, gives insight that is complementary to that obtained with the counting statistics [17].

The counting statistics of other transport dark-state models such as the triple quantum dot have previously been reported [18-25], with the dark state generally leading to super-Poissonian statistics associated with electron bunching. For the CNT-QD model here, we also obtain super-Poissonian statistics. However, the degree of this effect depends very strongly on a parameter $\Delta \phi$ which describes the phase difference between tunneling states of the source and drain leads. Indeed, for small $\Delta \phi$, we report a diverging noise and a skewness that both diverges and changes sign. In this regard, we conclude that the behavior of the model is similar to that of the Aharonov-Bohm interferometer models discussed in Refs. [26,27]. We also study in detail the effect on the counting statistics of two mechanisms that break the coherent population trapping, namely, relaxation and a Lamb-shift precession.

Concerning the waiting-time distribution, we show that the presence of the dark state gives rise to distributions with extremely long tails. Moreover, under certain conditions, we find that the waiting-time distribution shows oscillations when the Lamb shift is the dominant dark-state unblocking mechanism. This gives, in principle at least, a means though which the dark-state-breaking mechanism could be identified.

Finally, by using the parameters and voltage dependence of the Lamb shifts from the CNT-QD experiment of Ref. [5], we outline how the above features would appear in this experiment. We discuss how noise measurements can be useful in estimating critical model parameters, especially $\Delta \phi$, and provide an additional test of whether dark-state physics is indeed responsible for the current rectification in the experiment of Donarini et al.

\section{MODEL}

Our starting point is the model derived by Donarini et al. to describe transport within the $N=0,1$ sector of the CNT-QD spectrum [5]. This sector consists of the many-body ground state $|0\rangle$ and two degenerate states $| \pm l\rangle$, each with a single excess electron of longitudinal orbital momentum $\pm l$. The spin of the electron serves only to provide degeneracy factors and is otherwise neglected here. The Coulomb blockade [28] prevents the system from being occupied by more than one excess electron in the bias window considered.

In a high-bias regime, electrons tunnel into the CNT-QD from the left and out to right (see Fig. 1). The respective tunneling rates are $4 \Gamma_{L}$ and $\Gamma_{R}$, with the factor of 4 coming from degeneracy. Due to off-diagonal elements in the tunnel coupling, electrons do not tunnel directly into or out of states $| \pm l\rangle$, but rather into and out of superpositions of them. Crucially, the relevant basis for tunneling out of the dot consists 


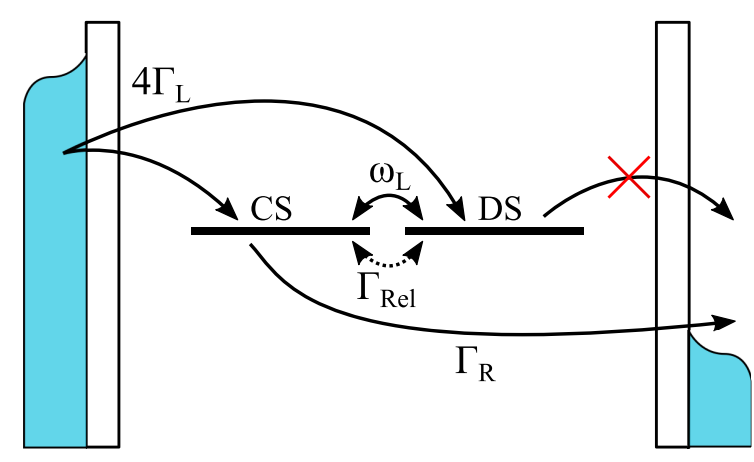

FIG. 1. Sketch of the CNT-QD transport model. Electrons enter the system from the left (source) lead with total rate $4 \Gamma_{L}$ into either the coupled state (CS) or dark state (DS). Electrons exit the system into the right (drain) lead at rate $\Gamma_{R}$ from only the CS. Without anything further, an electron entering the dark state thus becomes trapped, blocking current flow. However, Lamb-shift precession (frequency $\omega_{L}$ ) and relaxation (rate $\Gamma_{\text {Rel }}$ ) transfer electrons between DS and CS, unblocking the system and leading to current flow.

of the states

$$
\begin{aligned}
|\mathrm{CS}\rangle & \equiv \frac{1}{\sqrt{2}}\left(e^{i \phi_{R}}|l\rangle+e^{-i \phi_{R}}|-l\rangle\right), \\
|\mathrm{DS}\rangle & \equiv \frac{1}{\sqrt{2}}\left(e^{i \phi_{R}}|l\rangle-e^{-i \phi_{R}}|-l\rangle\right),
\end{aligned}
$$

where phase $\phi_{R}$ is a parameter characterizing the coupling to the right (drain) lead. Here CS denotes the "coupled state," and electrons in this state can leave the CNT-QD to the drain. Conversely, DS stands for "dark state," and this state is decoupled from the drain lead such that electrons entering it cannot tunnel out. There exists a similar basis for tunneling into the CNT-QD from the left lead. This is of the same form but with parameter $\phi_{L}$ instead of $\phi_{R}$.

The current blocking by the dark state is then driven by the overlap of these two sets of states, and this is governed by the phase difference $\Delta \phi=\phi_{L}-\phi_{R}$. When $\Delta \phi=0$, the bases for tunneling through left and right leads are the same. Thus, electrons tunnel from the left into the coupled state $|\mathrm{CS}\rangle$ and then tunnel directly out to the right. There is then no dark-state effect in the current flow. However, when $\Delta \phi \neq 0$, the left and right tunneling bases are different, and electrons from the left tunnel into both coupled and dark states. Once they enter the dark state, electrons cannot tunnel out of it and thus remain permanently trapped, and current is blocked.

Aside from tunneling, two further mechanisms are taken into account in the model, both of which serve to unblock the dark state. The first is the precession of the internal states, arising from Lamb shifts due to the coupling of the leads. In Ref. [5], the frequencies $\omega_{L}$ and $\omega_{R}$ of these shifts were found to be functions of applied voltages. In Secs. III-V, we take them as freely adjustable parameters to explore the model and set $\omega_{R}=\omega_{L}$ for convenience. In Sec. VI, we consider the voltage dependence of these quantities. The second unblocking mechanism is relaxation caused by inelastic processes such as phonon emission and absorption. This drives the internal state of the CNT-QD into the completely mixed state with a rate $\Gamma_{\text {Rel }}$.

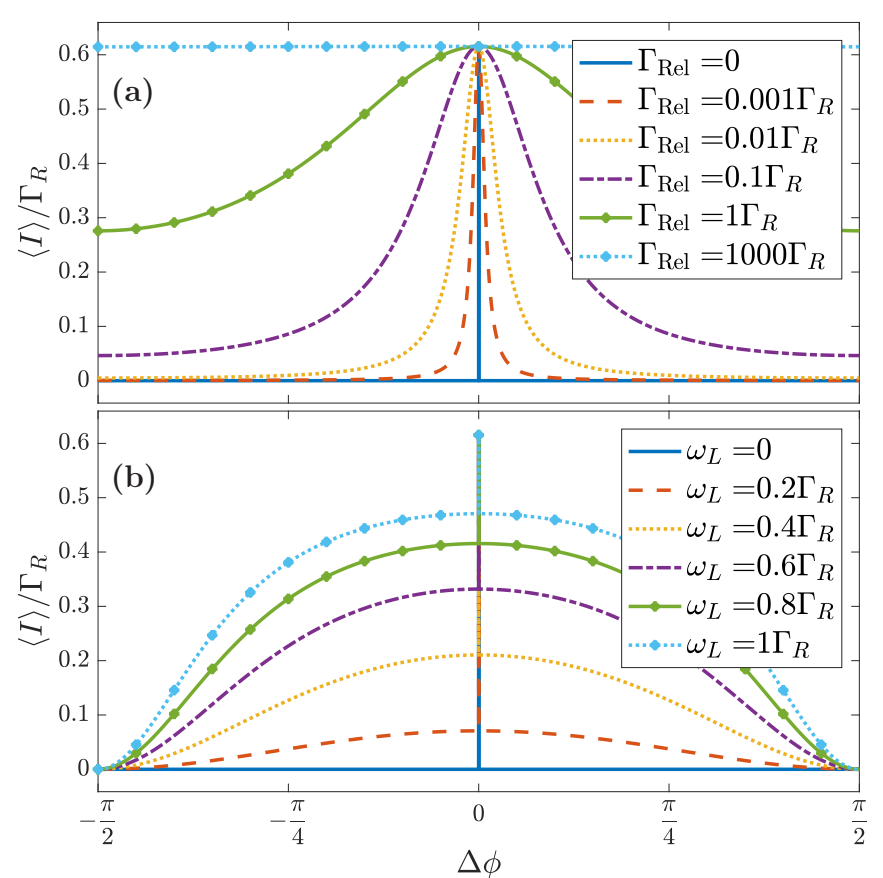

FIG. 2. Mean current $\langle I\rangle$ of the CNT-QD as a function of the tunnel-basis phase difference $\Delta \phi$ with $\Gamma_{L}=0.4 \Gamma_{R}$. (a) shows the effect of increasing relaxation rate $\Gamma_{\text {Rel }}$ with $\omega_{L}=0$. (b) shows the effect of increasing precession frequency $\omega_{L}$ with $\Gamma_{\text {Rel }}=0$. With $\omega_{L}=\Gamma_{\text {Rel }}=0$ the current is exactly zero for all $\Delta \phi \neq 0$ as the DS blockade is complete. Increasing the strength of either of the unblocking interactions increases the current with the largest values for $\Delta \phi \rightarrow 0$.

In the weak-coupling regime, the transport properties of this system can be determined from a quantum master equation of the form

$$
\dot{\rho}=\mathcal{W} \rho,
$$

where $\rho$ is the reduced density matrix of the CNT-QD and $\mathcal{W}$ is the Liouville superoperator describing all relevant dynamical processes. Appendix A shows the Liouvillian for the problem at hand in matrix form. In Appendix B we outline the counting-statistics formalism for calculating the cumulants of the current $\left\langle I^{k}\right\rangle_{c}$ (for $k=1,2,3$ here) as well as the waitingtime distribution $w(\tau)$ from master equation (2).

\section{CURRENT}

The impact of the DS on transport through the CNT-QD is immediately seen in the mean current $\langle I\rangle$. The results we obtain are consistent with those of Ref. [5], but here we separate out the influence of the two unblocking mechanisms.

Figure 2 shows the mean current as a function of the phase difference between tunneling states of the left and right leads, $\Delta \phi \equiv \phi_{L}-\phi_{R}$. For any nonzero phase difference, electrons have a finite probability of tunneling into the DS. In the absence of unblocking mechanisms, this state is decoupled from the right lead, and an electron entering it becomes permanently trapped. This in turn results in complete current suppression since no further electrons may tunnel into the system due to the Coulomb blockade. 
This entrapment is lifted, however, by the relaxation and precession mechanisms. A finite relaxation rate allows for electrons to move from the DS to the CS and then escape into the right lead. Similarly, the Lamb-shift precession causes electrons to oscillate between the CS and DS at a frequency of $\omega_{L}$, and this allows electrons to escape. As seen in Fig. 2, increasing the strength of either of these processes results in less suppression. The efficacy of the precession mechanism in unblocking the system is dependent on the phase difference.

Two special points are evident from these graphs. The first is $\Delta \phi=0$, where, in the absence of relaxation, the DS is completely decoupled from both the left and right leads. In this case, the part of the system involved in transport is essentially a single-level system. The counting statistics of this model are then fully known, as recounted in Appendix C. The second special point occurs at $\Delta \phi=\pi / 2$, where electrons from the left lead tunnel directly into the DS. With unblocking mechanisms present, the maximum current always occurs at $\Delta \phi=0$, and the minimum always occurs at $\Delta \phi=\pi / 2$. Furthermore, at these points, the current is unchanged by the precession frequency.

\section{SHOT NOISE AND SKEWNESS}

The second and third current cumulants are the shot noise and skewness, respectively. It is often more useful to discuss the cumulants in terms of their Fano factors, defined as the ratio of the $k$ th cumulant to the first: $F_{k}=\left\langle I^{k}\right\rangle_{c} /\langle I\rangle$. The second Fano factor is often just referred to as the Fano factor.

Figure 3 shows the (shot noise) Fano factor $F_{2}$ as a function of the phase difference for a range of relaxation rates and
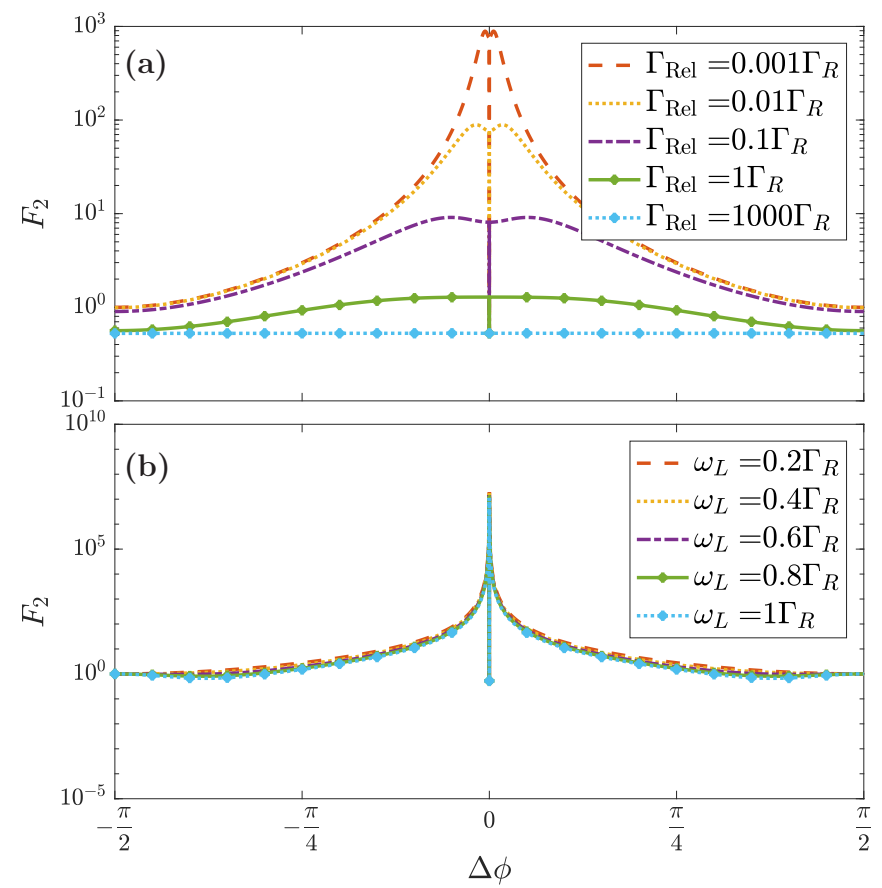

FIG. 3. Shot noise Fano factor $F_{2}$ as a function of the phase difference $\Delta \phi$ for (a) increasing relaxation rate $\Gamma_{\text {Rel }}$ with $\omega_{L}=0$ and (b) increasing precession frequency $\omega_{L}$ with $\Gamma_{\text {Rel }}=0$. The most striking feature here is the giant super-Poissonian values assumed by the Fano factor as $\Delta \phi \rightarrow 0$. Tunnel rates are set as $\Gamma_{L}=0.4 \Gamma_{R}$.

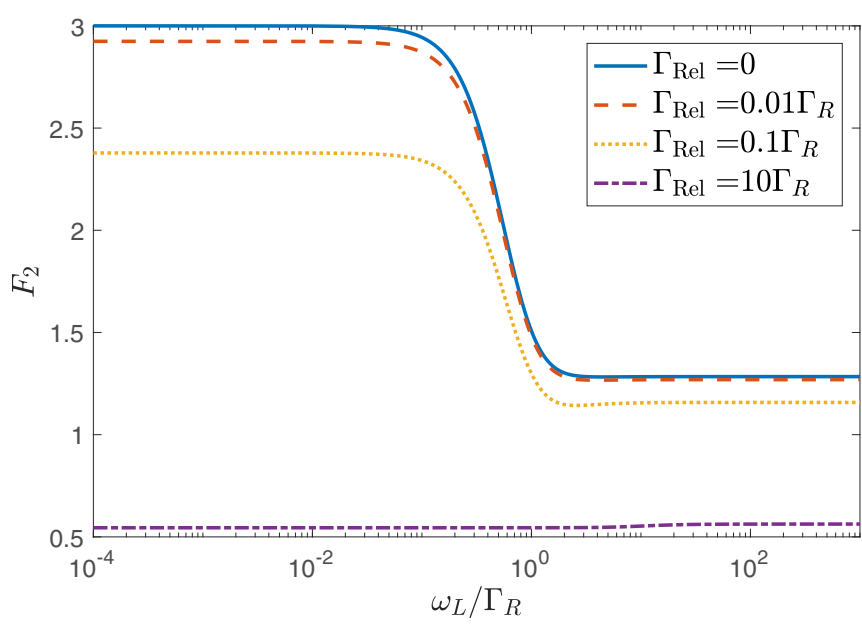

FIG. 4. The shot noise Fano factor of the CNT-QD as a function of the precession frequency with varying relaxation rate $\Gamma_{\text {Rel }}$. Parameters were $\Gamma_{L}=0.4 \Gamma_{R}$ and $\Delta \phi=\pi / 4$. At low relaxation, the Fano factor shows a transition from low to high values as the precession frequency is decreased. For both low precession frequency and relaxation rate, the Fano factor tends to a value of $F_{2}=3$ here. With a high relaxation rate, the system is sub-Poissonian for all precession frequencies.

precession frequencies. Figure 3(a) shows that $F_{2}$ increases as relaxation decreases for all values of the phase difference. The most striking thing about this plot is that, provided $\Gamma_{\text {Rel }} \lesssim \Gamma_{R}$ and the phase difference is not near $\pm \pi / 2$, the Fano factor assumes a value way in excess of the Poisson value $F_{2}=1$. And, indeed, as both $\Delta \phi \rightarrow 0$ and $\Gamma_{\mathrm{Rel}} \rightarrow 0$, the Fano factor is observed to diverge.

Figure 3(b) shows the effect on $F_{2}$ of changing the precession frequency. Once again, for finite $\omega_{L}$, giant superPoissonian values of $F_{2}$ are observed, with divergence occurring for $\Delta \phi \rightarrow 0$. For values of $\Delta \phi$ away from the origin, the change in $F_{2}$ is less drastic than in the case with changing $\Gamma_{\text {Rel. }}$. In Fig. 4 we plot $F_{2}$ as a function of precession frequency for several relaxation rates with a fixed phase difference of $\Delta \phi=\pi / 4$, far away from the diverging limit. Figure 4 shows that, provided $\Gamma_{\text {Rel }}$ is small enough, as the precession frequency is decreased, the Fano factor undergoes a transition from a value close to 1 to a value significantly in excess of it (here $F_{2} \rightarrow 3$ as $\omega_{L} \rightarrow 0$ for $\Gamma_{\text {Rel }}=0$ ). This transition to the higher $F_{2}$ value is an indication that the blocking of the DS is starting to play a significant role. For large $\Gamma_{\text {Rel }}$ the statistics are sub-Poissonian irrespective of $\omega_{L}$. This is as expected because under such circumstances the internal quantum structure of the system becomes irrelevant and the system essentially becomes a single (degenerate) level system.

The third (skewness) Fano factor is plotted in Fig. 5 as a function of $\Delta \phi$. Once again, we observe giant superPoissonian values, even larger than those seen with $F_{2}$ for the same parameters. As $\Delta \phi \rightarrow 0, F_{3}$ becomes negative and for $\Gamma_{\text {Rel }} \rightarrow 0$ or $\omega_{L} \rightarrow 0$ diverges as $F_{3} \rightarrow-\infty$.

\section{EFFECTIVE MODELS}

We now discuss two effective models that allow us to explain many of the features of the foregoing results, as 

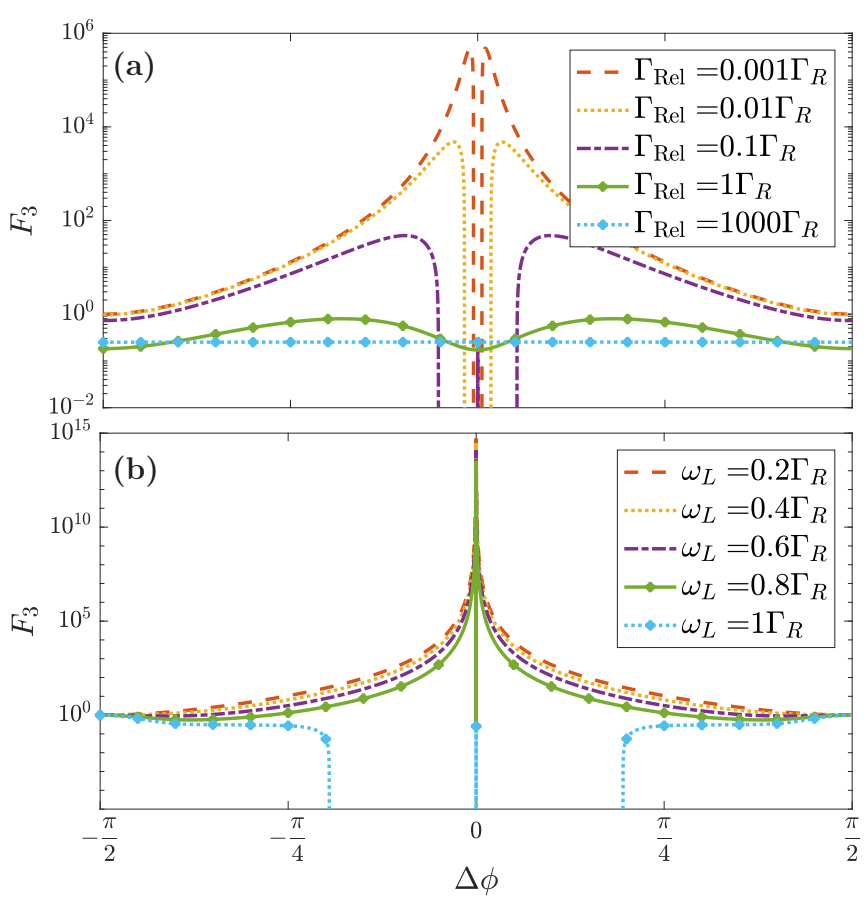

FIG. 5. The same as Fig. 3, but here we plot the skewness Fano factor $F_{3}$. As is the case for $F_{2}$, the third Fano factor is massively super-Poissonian. In addition, for $\Delta \phi \rightarrow 0, F_{3}$ becomes negative (which translates as the curves leaving the bottom of these logarithmic plots).

well as to connect with previous studies in the literature. We concentrate on the $\omega_{L}=0$ case with relaxation being the dominant unblocking mechanism.

Instead of using the quantum master equation, Eq. (2), we can alternatively describe the system with a rate equation involving the populations of the three states $|0\rangle,|\mathrm{CS}\rangle$, and $|\mathrm{DS}\rangle$. In this picture, electrons tunnel into the CS with a rate $4 \Gamma_{L} \cos ^{2}(\Delta \phi)$ and into the DS with a rate $4 \Gamma_{L} \sin ^{2}(\Delta \phi)$. Without relaxation, tunneling to the right lead occurs only from the CS with rate $\Gamma_{R}$. However, when $\Gamma_{\text {Rel }}$ is finite, electrons can leave the system by first relaxing into the coupled state and then tunneling out. When $\Gamma_{\text {Rel }} \ll \Gamma_{R}$, the speed of this process is limited by the relaxation step, and we can write this unblocking step as an effective out tunneling from the DS to state $|0\rangle$ at a rate $\Gamma_{\text {eff }} \approx \Gamma_{\text {Rel }}$. Thus, we describe the system with a $\chi$-resolved rate equation $\dot{P}=\mathcal{W}(\chi) P$, where

$$
\mathcal{W}(\chi)=\left(\begin{array}{ccc}
-4 \Gamma_{L} & \Gamma_{R} e^{i \chi} & \Gamma_{\mathrm{eff}} e^{i \chi} \\
4 \Gamma_{L} \cos ^{2}(\Delta \phi) & -\Gamma_{R} & 0 \\
4 \Gamma_{L} \sin ^{2}(\Delta \phi) & 0 & -\Gamma_{\mathrm{eff}}
\end{array}\right)
$$

and where, in the $\chi \rightarrow 0$ limit, $P$ is the vector of populations of the $|0\rangle,|\mathrm{CS}\rangle$, and $|\mathrm{DS}\rangle$ states.

With this simplified model, exact expressions for the current cumulants are possible. Reporting results in the large- $\Gamma_{R}$ limit, we obtain

$$
\langle I\rangle=\frac{4 \Gamma_{\mathrm{eff}} \Gamma_{L} \csc (\Delta \phi)^{2}}{4 \Gamma_{L}+\Gamma_{\mathrm{eff}} \csc (\Delta \phi)}
$$

and

$$
F_{2}=\frac{\Gamma_{\mathrm{eff}}^{2}+10 \Gamma_{L}^{2}-2 \Gamma_{L}^{2}[4 \cos (2 \Delta \phi)+\cos (4 \Delta \phi)]}{\left[\Gamma_{\text {eff }}+2 \Gamma_{L}-2 \Gamma_{L} \cos (2 \Delta \phi)\right]^{2}} .
$$

The corresponding expression for the skewness is rather cumbersome and not especially illuminating. Good agreement is found between the full numerics and these expressions in the appropriate regime.

This effective model then permits us to make an immediate connection with the dynamical channel blockade models of Belzig and coworkers [29,30]. Indeed, with $\Delta \phi=\pi / 4$ as in Fig. 4, the rates of tunneling into each of the CS and DS become equal, and the model here becomes identical to that of Ref. [30]. This then explains the Fano factor value of $F_{2}=3$ in the limit $\omega_{L} \rightarrow 0$ for $\Gamma_{\mathrm{Rel}}=0$ as arising from electron bunches with multiples of three electrons per bunch. This then also matches with the Fano factor found by Groth et al. [18] for the triple-quantum-dot model. For $\Delta \phi \neq \pi / 4$, the tunnel rates into the two states become unequal, and this then significantly changes the bunching properties of the current flow.

Our second effective model provides a simple explanation of the diverging Fano factors, as well as their signs. For $\Gamma_{\text {Rel }} \approx 0$, our transport system is essentially bistable [31] in one of its steady states (the DS) the system does not conduct; in the other, it does and admits a mean current, $\langle I\rangle_{0}$, say. The probability distribution for the number of transferred charges will therefore be approximately $P(n, t)=$ $(1-p) \delta_{n, 0}+p \delta_{n, n_{0}}$, where $p$ is the probability that we find ourselves in the conducting channel and $n_{0}=\langle I\rangle_{0} t$ is the mean number of transferred charges in time $t$ if we do. If $t$ is large, we are justified in ignoring the small fluctuations in the charge numbers of zero and $n_{0}$. The cumulant generating function for this model reads $\mathcal{F}(\chi, t)=\ln \left(1-p+p e^{i n_{0} \chi}\right)$, which means we have a Bernoulli distribution with "payoff" $n_{0}$. The mean current is $\langle I\rangle=t^{-1} \partial \mathcal{F} /\left.(\partial(i \chi))\right|_{0}=p\langle I\rangle_{0}$, which is the mean current of the conducting state multiplied by the probability of obtaining that state. Then, the first two Fano factors read $F_{2}=\langle I\rangle_{0} t(1-p)$ and $F_{3}=\langle I\rangle_{0}^{2} t^{2}(1-p)(1-2 p)$. In the asymptotic limit, $t \rightarrow \infty$, the Fano factors diverge as $F_{k} \sim t^{k-1}$. Moreover, while the sign of $F_{2}$ is manifestly positive (since $1-p>0$ ), the skewness will be positive for $p<1 / 2$ and negative for $p>1 / 2$ due to the factor $(1-2 p)$. Indeed, this simple model suggests that negative skewness is associated with a bistable situation when the probability of finding the conducting channel is large and that the skewness will transfer to being positive as the weight of the blocking channel increases. This is the behavior observed in the DS model here.

\section{WAITING-TIME DISTRIBUTION}

The waiting-time distribution $\omega(\tau)$ gives the probability of waiting a time $\tau$ between consecutive "jumps" of an electron out of the system. Figure 6 shows the waiting-time distribution for the same parameters as Fig. 2(a) with the phase difference fixed at $\Delta \phi=\pi / 4$. While on a linear scale the distribution $w(\tau)$ looks similar to that which would be obtained from a single-level quantum dot [13], on a log scale (Fig. 6, inset), we see that the distribution possesses an extremely long tail, and 


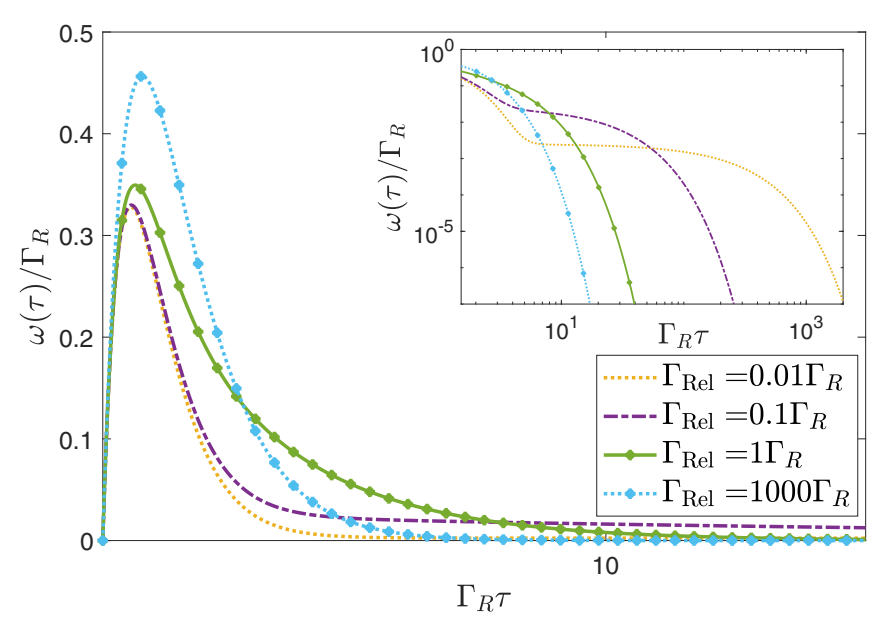

FIG. 6. Waiting-time distributions for various $\Gamma_{\text {Rel }}$ with $\omega_{L}=0$, $\Gamma_{L}=0.4 \Gamma_{R}$, and $\Delta \phi=\pi / 4$. The inset shows the same data on a logarithmic scale, which highlights the long tail of the distribution induced by the dark state.

the lower the relaxation rate is, the longer the tail becomes. This tail is due to the presence of the DS, which results in an electron being trapped for a long time before exiting the system. The separation of timescales can be extreme. For $\Gamma_{\mathrm{Rel}} / \Gamma_{R}=0.01$, for example, the main peak of the tunneling dynamics is over after $\tau \approx 5 \Gamma^{-1}$, whereas the bulk of the tail extends out to a time of $\tau \approx 10^{3} \Gamma^{-1}$. We note that the waitingtime distribution shows no particular trace of the change in sign of the third Fano factor.

Figure 7 shows the waiting-time distribution when the precession frequency is finite. In the regime when $\Gamma_{L}>\Gamma_{R}$, the precession of the electrons oscillating between the CS and DS imprints oscillations on the waiting-time distribution. As the precession frequency increases, the observed oscillation frequency increases, but the amplitude decreases. Under these conditions, the waiting-time distribution still

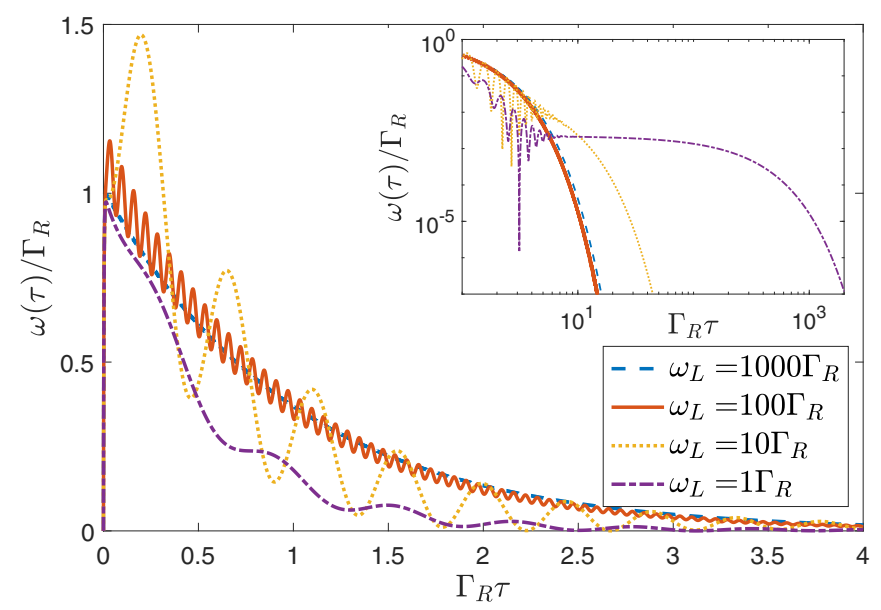

FIG. 7. Waiting-time distributions for $\Gamma_{\text {Rel }}=0, \Gamma_{L}=100 \Gamma_{R}$, and $\Delta \phi=\pi / 4$ on both linear and logarithmic scales. For these parameters, oscillations due to the Lamb-shift precessions are clearly visible. maintains the extended tail, indicating the continued influence of the DS.

\section{EXPERIMENTAL SIGNATURES}

We now consider the appearance of some of the above features in the experimental setup of Ref. [5]. In the gated CNT-QD setup, the Lamb shifts are determined by the applied bias $V_{B}$ and gate $V_{G}$ voltages via [5] $(e=1)$

$$
\omega_{\alpha}=\frac{\Gamma_{\alpha}}{\pi}\left[p_{\alpha}\left(-V_{G}\right)-p_{\alpha}\left(U-\frac{J}{2}-V_{G}\right)\right],
$$

with

$$
p_{\alpha}(E)=-\operatorname{Re} \psi\left[1 / 2+i\left(E-\mu_{\alpha}\right) /\left(2 \pi k_{B} T\right)\right] .
$$

Here $\psi$ is the digamma function, $U$ is the QD charging energy, $J$ is the exchange interaction strength, and $k_{B} T$ is the thermal energy. The chemical potentials of the leads are set as $\mu_{L}=-\eta V_{B}$ on the left and $\mu_{R}=(\eta-1) V_{B}$ on the right, with $\eta$ being a parameter to account for an asymmetric bias drop at the two leads.

The model we have hitherto considered is valid for large reverse bias, specifically, when $\left|V_{B}\right|$ is larger than all other relevant energy scales, i.e., $k_{B} T, \hbar \omega_{\alpha}$, etc., and also $\eta V_{B}<V_{G}<$ $(\eta-1) V_{B}$ such that transitions lie within the bias window. The corresponding model for forward bias can be obtained by swapping all "left" and "right" quantities in Liouvillian (A1). This forward-bias calculation is then valid at high bias with $(\eta-1) V_{B}<V_{G}<\eta V_{B}$.

Figure 8(a) shows the mean current through the CNTQD as a function of gate voltage for both forward- and reverse-bias configurations. We plot results for a typical value of $V_{B}= \pm 3 \mathrm{mV}$ and for three different phase differences: $\Delta \phi=(0.5,1,2) \times \Delta \phi_{\exp }$, where $\Delta \phi_{\exp }=0.11 \pi$ is the value extracted from experiment [5]. Our results for $\Delta \phi=\Delta \phi_{\exp }$ agree with those of Donarini et al. and show the rectification that arises from the combination of dark-state trapping and asymmetry in the coupling of the CNT to the leads.

Figure 8(b) shows the predicted shot noise Fano factor for the same parameters. We again see a clear distinction between forward- and reverse-bias results, with the reverse-bias Fano factor significantly in excess of that with forward bias. For the experimentally determined phase difference, $\Delta \phi=\Delta \phi_{\text {exp }}$, the Fano factor at $V_{G}=0$ is strongly super-Poissonian, $F_{2}=$ 15.5, showing the effect of the dark state. In forward bias, the $V_{G}=0$ value is also super-Poissonian, $F_{2}=5.0$, albeit reduced relative to the reverse-bias value. This difference reinforces that the dark-state trapping is more active in reverse than forward bias.

More significant than the exact value of $F_{2}$ is its sensitivity to changes in the phase difference $\Delta \phi$. Reference [5] states that experimentally, most of the model parameters can be extracted from the Coulomb diamond properties, and of those that cannot, the phase difference $\Delta \phi$ and the ratio $\Gamma_{L} / \Gamma_{R}$ (for fixed total rate $\Gamma_{L}+\Gamma_{R}$ ) have the greatest effect in determining the current. Figure 8(a) shows, however, that changing $\Delta \phi$ by a factor of 2 either way leads to only relatively small changes in the mean current. In contrast, the Fano factor changes dramatically with a change in $\Delta \phi$, as can be seen 

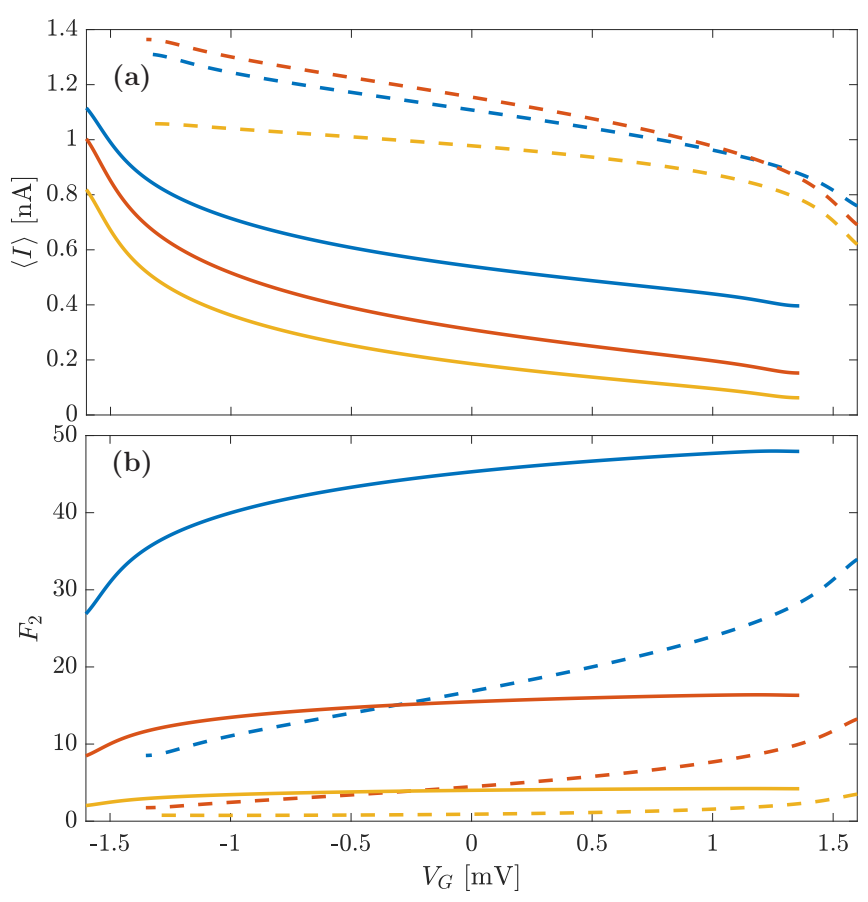

FIG. 8. (a) Mean current and (b) shot noise Fano factor $F_{2}$ for the CNT-QD experiment as a function of gate voltage $V_{G}$. Results are shown for three values of the phase difference $\Delta \phi / \Delta \phi_{\exp }=0.5,1,2$ (blue, red, and yellow curves, respectively), where $\Delta \phi_{\exp }=0.11 \pi$ is the value found in experiment. Solid lines show reverse-bias results; dashed lines are those for forward bias. Strong asymmetry in both current and noise is observed due to the difference in efficacy of the trapping mechanism in the two bias directions. The Fano factor is seen to be sensitive to changes in the phase difference $\Delta \phi$. The bias voltage was set as $V_{B}= \pm 3 \mathrm{mV}$, with other parameters taken from Ref. [5]: $U=20 \mathrm{meV}, J=10 \mu \mathrm{eV}, k_{B} T=50 \mu \mathrm{eV}, \Gamma_{L}=4 \mu \mathrm{eV}$, $\Gamma_{R}=10 \mu \mathrm{eV}, \Gamma_{\text {rel }}=0.1 \mu \mathrm{eV}$, and $\eta=0.55$.

from Fig. 8(b). At $V_{G}=0$ the reverse-bias Fano factor drops to $F_{2}=4.0$ at $\Delta \phi=2 \Delta \phi_{\exp }$ but increases to $F_{2}=45.3$ at $\Delta \phi=1 / 2 \Delta \phi_{\text {exp }}$. This dramatic change is due to the strong nonlinearity of the diverging Fano factor as described in Sec. IV.

This sensitivity of $F_{2}$, compared with that of the current, should mean that the addition of noise measurements will enable $\Delta \phi$ to be estimated more robustly from experiment than would be the case from current measurements alone.

In Fig. 9 we investigate the current and noise characteristics as a function of gate voltage with changes in the ratio of $\Gamma_{L} / \Gamma_{R}$ with total rate $\Gamma_{L}+\Gamma_{R}$ fixed. We find that both quantities change significantly with $\Gamma_{L} / \Gamma_{R}$ and that the dependence of $F_{2}$ on $\Gamma_{L} / \Gamma_{R}$ is less marked than in its $\Delta \phi$ dependence. The difference between forward and reverse properties with $\Gamma_{L} / \Gamma_{R}=1$ stems from the inclusion of asymmetry factor $\eta \neq 1 / 2$, such that a small degree of rectification persists in this limit.

Although the shot noise is the most readily accessible cumulant with current technology, we also plot in Fig. 10 the predicted skewness Fano factor $F_{3}$ as a function of gate voltage. As can be anticipated from the noise, the skewness Fano factor shows large values $\left(F_{3} \approx 275\right.$ at $\left.V_{G}=0\right)$ for experimentally relevant parameters, and the results are very
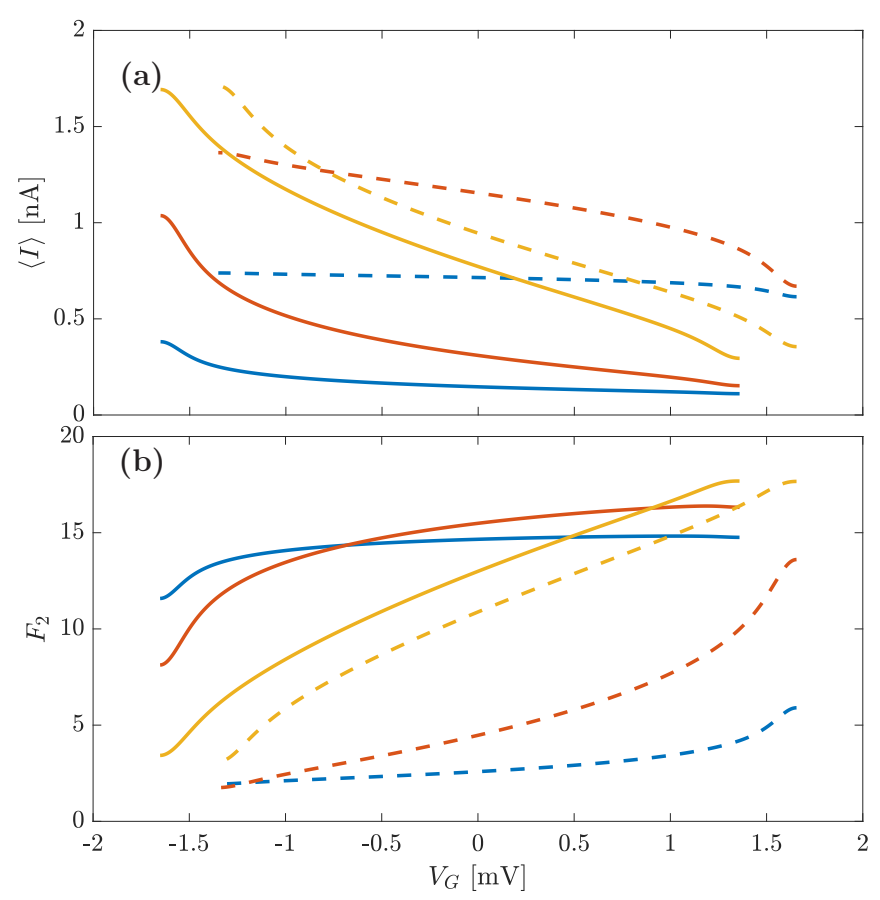

FIG. 9. The same as Fig. 8, but here we plot results for different values of $\Gamma_{L}=2,4,7 \mu \mathrm{eV}$ (blue, red, and yellow curves, respectively), with the total rate $\Gamma_{L}+\Gamma_{R}=14 \mu \mathrm{eV}$ fixed at the experimentally observed value. Significant dependence of both quantities on the ratio $\Gamma_{L} / \Gamma_{R}$ is observed, but not as marked as the $\Delta \phi$ dependence.

sensitive to small changes in the phase difference $\Delta \phi$. Also of interest is that, for the parameters found in Donarini et al., the forward-bias skewness is predicted to be negative throughout large parts of the gate-voltage range.

\section{DISCUSSION}

In summary we have calculated the first three current cumulants of a CNT-QD containing a dark state. These cumulants are characterized by giant super-Poissonian Fano factors brought about by electron bunching induced by the dark state. The Fano factors show a strong dependence on

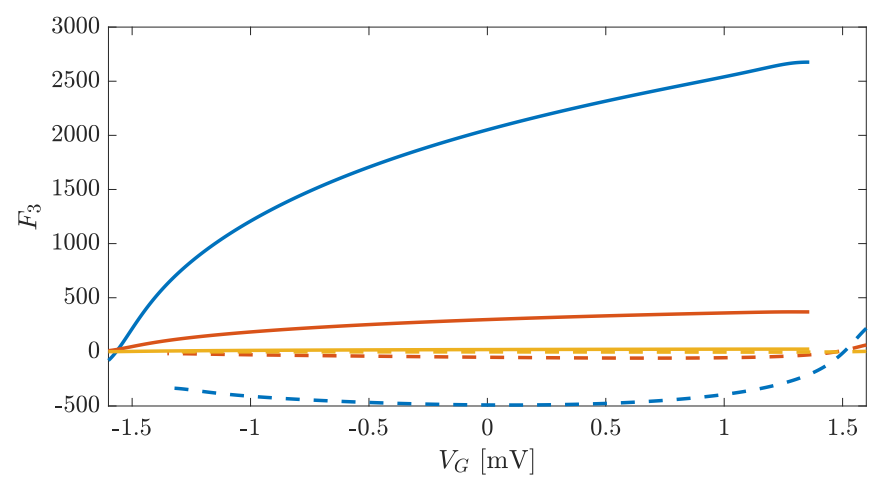

FIG. 10. Skewness Fano factor $F_{3}$ for the CNT-QD setup as a function of gate voltage $V_{G}$. Parameters and the legend are the same as in Fig. 8. With reverse bias, large positive values of $F_{3}$ are observed, whereas at forward bias, the skewness is negative across large portions of the gate-voltage range. 
the phase difference $\Delta \phi$, and for $\Delta \phi \rightarrow 0$ with vanishing relaxation, the Fano factors diverge with $F_{2}>0$ and $F_{3}<0$. This behavior can be explained by noting that, in this limit, the system is essentially bistable with a very long (in the limit, diverging) switching time between the states. The phase dependence, including the periodic divergence of the Fano factors, is very similar to that reported Urban and König [26] (see also Li et al. [27]) for an Aharanov-Bohm interferometer with quantum dots in the arms. In that context, the decisive phase is the flux through the interferometer, and as the flux varies, quantum dot states couple and decouple from the leads, similar to the behavior of the dark state here.

The bunching and bistability caused by the dark state are also responsible for giving the waiting-time distributions the characteristic form found here. These are composed of an initial peak, corresponding to the conducting channel, followed by an extensive tail corresponding to long times that the system spends trapped in the dark state.

Using the parameters obtained for the CNT-QD measured in Ref. [5], we predict that large Fano factors (of the order of $F_{2} \approx 16$ and $F_{3} \approx 275$ ) should be observable in experiment, with a marked dependence on bias direction. We also predict that both signs should be apparent in the measurement of the skewness Fano factor. The predicted sensitivity of the noise Fano factor means that its measurement will be able to more robustly determine the critical parameter $\Delta \phi$. Finally, we point out that measurements of noise, and higher-order statistics, provide a more detailed test of the mechanisms at play in electron transport $[12,16]$, and comparison with the predictions made here might reveal the need for modifications to this dark-state model of CNT-QD transport.

\section{ACKNOWLEDGMENTS}

The authors would like to thank A. Donarini and M. Grifoni for helpful discussions.

\section{APPENDIX A: CNT-QD LIOUVILLIAN}

For the CNT-QD model, the density matrix has five relevant entries, which we organize into the vector $|\rho\rangle\rangle=$ $\left(\rho_{00}, \rho_{l l}, \rho_{-l-l}, \rho_{l-l}, \rho_{-l l}\right)^{\mathrm{T}}$, with transpose T. In this basis, the Liouvillian for our problem reads

$$
\mathcal{W}(\chi)=\left(\begin{array}{ccccc}
-4 \Gamma_{L} & \Gamma_{R} e^{i \chi} & \Gamma_{R} e^{i \chi} & e^{-2 i \phi_{R}+i \chi} \Gamma_{R} & e^{2 i \phi_{R}+i \chi} \Gamma_{R} \\
2 \Gamma_{L} & -\Gamma_{R}-\frac{\Gamma_{\mathrm{Rel}}}{2} & \frac{\Gamma_{\mathrm{Rel}}}{2} & -\left(\frac{\Gamma_{R}}{2} e^{-2 i \phi_{R}}-i \tilde{\omega}^{*}\right) & -\left(\frac{\Gamma_{R}}{2} e^{2 i \phi_{R}}+i \tilde{\omega}\right) \\
2 \Gamma_{L} & \frac{\Gamma_{\mathrm{Rel}}}{2} & -\Gamma_{R}-\frac{\Gamma_{\mathrm{Rel}}}{2} & -\left(\frac{\Gamma_{R}}{2} e^{-2 i \phi_{R}}+i \tilde{\omega}^{*}\right) & -\left(\frac{\Gamma_{R}}{2} e^{2 i \phi_{R}}-i \tilde{\omega}\right) \\
2 \Gamma_{L} e^{2 i \phi_{L}} & -\left(\frac{\Gamma_{R}}{2} e^{2 i \phi_{R}}-i \tilde{\omega}\right) & -\left(\frac{\Gamma_{R}}{2} e^{2 i \phi_{R}}+i \tilde{\omega}\right) & -\left(\Gamma_{R}+\Gamma_{\mathrm{Rel}}\right) & 0 \\
2 \Gamma_{L} e^{-2 i \phi_{L}} & -\left(\frac{\Gamma_{R}}{2} e^{-2 i \phi_{R}}+i \tilde{\omega}^{*}\right) & -\left(\frac{\Gamma_{R}}{2} e^{-2 i \phi_{R}}-i \tilde{\omega}^{*}\right) & 0 & -\left(\Gamma_{R}+\Gamma_{\mathrm{Rel}}\right)
\end{array}\right),
$$

where $\tilde{\omega}=\omega_{L} e^{2 i \phi_{L}}+\omega_{R} e^{2 i \phi_{R}}$. Anticipating Appendix B, we include here the counting-field factor $e^{i \chi}$ on all tunnel terms to the right lead.

\section{APPENDIX B: COUNTING STATISTICS METHOD}

Here we follow the full counting statistics formalism for Markovian master equations as described in, e.g., Refs. [8,32$35]$. With the relevant density-matrix elements written into length- $N$ vector $|\rho(t)\rangle\rangle$, master equation (2) can be written as $\left.\left.\frac{d}{d t}|\rho(t)\rangle\right\rangle=\mathcal{W}|\rho(t)\rangle\right\rangle$, where $\mathcal{W}$ is the Liouville superoperator in matrix form. Transport is then described by the $\chi$-resolved master equation

$$
\left.\left.\frac{d}{d t}|\rho(\chi, t)\rangle\right\rangle=\mathcal{W}(\chi)|\rho(\chi, t)\rangle\right\rangle,
$$

where $\mathcal{W}(\chi)=\mathcal{W}_{0}+e^{i \chi} \mathcal{J}$ is the Liouvillian decomposed into jump $(\mathcal{J})$ and nonjump $\left(\mathcal{W}_{0}\right)$ parts and where $\chi$ is the counting field. We have $\lim _{\chi \rightarrow 0} \mathcal{W}(\chi)=\mathcal{W}=\mathcal{W}_{0}+\mathcal{J}$. The stationary state of the system is given by $\left.\mathcal{W}\left|\rho_{\text {stat }}\right\rangle\right\rangle=0$, which we assume to be unique. The left null vector of $\mathcal{W}$, denoted $\left\langle\langle 1|\right.$, is normalized such that $\left\langle\left\langle\mathbb{1} \mid \rho_{\text {stat }}\right\rangle\right\rangle=1$. Multiplication with this vector corresponds to taking the trace of the density matrix. The expectation value of a general Liouville space operator $\mathcal{A}$ acting on state $\rho$ is thus given by $\langle\langle\mathcal{A}\rangle\rangle=\left\langle\left\langle\mathbb{1}|\mathcal{A}| \rho_{\text {stat }}\right\rangle\right\rangle$. We also define $\left.\mathcal{P}=\left|\rho_{\text {stat }}\right\rangle\right\rangle\langle\langle\mathbb{1}|$ as the stationary-state projection matrix and $\mathcal{R}$ as the pseudoinverse of $\mathcal{W}$.

The generating function of the cumulants of transferred charge is given by $\mathcal{F}(\chi, t)=\lambda_{0}(\chi) t$, where $\lambda_{0}$ is that eigenvector of $\mathcal{W}(\chi)$ which reverts to zero in the $\chi \rightarrow 0$ limit. The current cumulants are then given by

$$
\left\langle I^{k}\right\rangle_{c}=\left.\frac{1}{t} \frac{\partial^{k}}{\partial(i \chi)^{k}} \mathcal{F}(\chi, t)\right|_{0} .
$$

Practically, however, finding the eigenvalues of $\mathcal{W}(\chi)$ is challenging for all but the simplest models, and an alternative approach is to expand the generating function to obtain explicit expressions for a finite set of cumulants. The expressions for the first three current cumulants read $(e=1)$

$$
\begin{aligned}
\langle I\rangle_{c}= & \langle\langle\mathcal{J}\rangle\rangle, \\
\left\langle I^{2}\right\rangle_{c}= & \langle\langle\mathcal{J}+2 \mathcal{J} \mathcal{R} \mathcal{J}\rangle\rangle \\
\left\langle I^{3}\right\rangle_{c}= & \langle\langle\mathcal{J}+3 \mathcal{J} \mathcal{R} \mathcal{J}+3 \mathcal{J} \mathcal{R} \mathcal{J} \\
& +6 \mathcal{J} \mathcal{R}[\mathcal{J} \mathcal{R}-\mathcal{R} \mathcal{J} \mathcal{P}] \mathcal{J}\rangle\rangle .
\end{aligned}
$$

The waiting-time distribution for a master equation can be expressed in this same language [13]. In the case of the carbon nanotube quantum dot we are concerned with only consecutive jumps of one type (transfer to the right lead), and the system is of the "single-reset" type. In this case, the 
waiting-time distribution is given by

$$
\omega(\tau)=\frac{\left\langle\left\langle\mathcal{J} e^{\mathcal{W}_{0} \tau} \mathcal{J}\right\rangle\right\rangle}{\langle\langle\mathcal{J}\rangle\rangle}
$$

\section{APPENDIX C: SINGLE-LEVEL MODEL AT $\Delta \phi=0$}

At $\Delta \phi=0$ and with $\Gamma_{\text {rel }}=0$, transport through the system can be described by the $\chi$-resolved Liouvillian

$$
\mathcal{W}(\chi)=\left(\begin{array}{cc}
-4 \Gamma_{L} & \Gamma_{R} e^{i \chi} \\
4 \Gamma_{L} & -\Gamma_{R}
\end{array}\right)
$$

written in the basis of empty state $|0\rangle$ and coupled state $|\mathrm{CS}\rangle$ populations. The resulting cumulant generating function is $[9,10]$

$$
\mathcal{F}(\chi)=\frac{\Gamma t}{2}\left(-1+\sqrt{a^{2}+4 \frac{\langle I\rangle}{\Gamma} e^{i \chi}}\right),
$$

in terms of the total rate $\Gamma=4 \Gamma_{L}+\Gamma_{R}$, asymmetry $a=$ $\left(4 \Gamma_{L}-\Gamma_{R}\right) / \Gamma$, and mean current

$$
\langle I\rangle=\frac{4 \Gamma_{L} \Gamma_{R}}{4 \Gamma_{L}+\Gamma_{R}} .
$$

The second and third Fano factors of this model read

$$
F_{2}=\frac{1+a^{2}}{2}, \quad F_{3}=\frac{1+3 a^{4}}{4} .
$$

Interestingly, whereas the current expression (C3) is also valid for $\Gamma_{\text {Rel }} \neq 0$, the higher cumulants differ significantly from the above.
[1] G. Alzetta, A. Gozzini, L. Moi, and G. Orriols, Nuovo Cimento B 36, 5 (1976).

[2] E. Arimondo and G. Orriols, Lett. Nuovo Cimento 17, 333 (1976).

[3] R. M. Whitley and C. R. Stroud, Phys. Rev. A 14, 1498 (1976).

[4] B. Michaelis, C. Emary, and C. Beenakker, Europhys. Lett. 73, 677 (2006).

[5] A. Donarini, M. Niklas, M. Schafberger, N. Paradiso, C. Strunk, and M. Grifoni, Nat. Commun. 10, 381 (2019).

[6] L. S. Levitov and G. B. Lesovik, Pis'ma Zh. Eskp. Teor. Fiz. 55, 534 (1992) [JETP. Lett. 55, 555 (1992)].

[7] L. S. Levitov, H. Lee, and G. B. Lesovik, J. Math. Phys. 37, 4845 (1996).

[8] D. A. Bagrets and Y. V. Nazarov, Phys. Rev. B 67, 085316 (2003).

[9] S. Gustavsson, R. Leturcq, B. Simovič, R. Schleser, T. Ihn, P. Studerus, K. Ensslin, D. C. Driscoll, and A. C. Gossard, Phys. Rev. Lett. 96, 076605 (2006).

[10] S. Gustavsson, R. Leturcq, T. Ihn, K. Ensslin, M. Reinwald, and W. Wegscheider, Phys. Rev. B 75, 075314 (2007).

[11] T. Fujisawa, T. Hayashi, and Y. R. Tomita, Science 312, 1634 (2006).

[12] A. Kurzmann, P. Stegmann, J. Kerski, R. Schott, A. Ludwig, A. D. Wieck, J. König, A. Lorke, and M. Geller, Phys. Rev. Lett. 122, 247403 (2019).

[13] T. Brandes, Ann. Phys. (Berlin, Ger.) 17, 477 (2008).

[14] L. S. Levitov and M. Reznikov, Phys. Rev. B 70, 115305 (2004).

[15] D. Loss and E. V. Sukhorukov, Phys. Rev. Lett. 84, 1035 (2000).

[16] G. Kießlich, E. Schöll, T. Brandes, F. Hohls, and R. J. Haug, Phys. Rev. Lett. 99, 206602 (2007).

[17] C. Emary, C. Pöltl, A. Carmele, J. Kabuss, A. Knorr, and T. Brandes, Phys. Rev. B 85, 165417 (2012).
[18] C. W. Groth, B. Michaelis, and C. W. J. Beenakker, Phys. Rev. B 74, 125315 (2006).

[19] C. Emary, Phys. Rev. B 76, 245319 (2007).

[20] C. Pöltl, C. Emary, and T. Brandes, Phys. Rev. B 80, 115313 (2009).

[21] I. Weymann, B. R. Bułka, and J. Barnaś, Phys. Rev. B 83, 195302 (2011).

[22] F. Domínguez, G. Platero, and S. Kohler, Chem. Phys. 375, 284 (2010).

[23] F. Domínguez, S. Kohler, and G. Platero, Phys. Rev. B 83, 235319 (2011).

[24] C. Pöltl, C. Emary, and T. Brandes, Phys. Rev. B 87, 045416 (2013).

[25] H.-K. Zhao, J. Wang, and Q. Wang, Phys. Lett. A 378, 1553 (2014).

[26] D. Urban and J. König, Phys. Rev. B 79, 165319 (2009).

[27] F. Li, H. J. Jiao, J. Y. Luo, X.-Q. Li, and S. A. Gurvitz, Phys. E (Amsterdam, Neth.) 41, 1707 (2009).

[28] D. V. Averin and K. K. Likharev, J. Low Temp. Phys. 62, 345 (1986).

[29] A. Cottet, W. Belzig, and C. Bruder, Phys. Rev. B 70, 115315 (2004).

[30] W. Belzig, Phys. Rev. B 71, 161301(R) (2005).

[31] G. Schaller, G. Kießlich, and T. Brandes, Phys. Rev. B 81, 205305 (2010).

[32] C. Flindt, T. Novotný, and A.-P. Jauho, Phys. Rev. B 70, 205334 (2004).

[33] A.-P. Jauho, C. Flindt, T. Novotný, and A. Donarini, Phys. Fluids 17, 100613 (2005).

[34] C. Flindt, T. Novotný, A. Braggio, M. Sassetti, and A.-P. Jauho, Phys. Rev. Lett. 100, 150601 (2008).

[35] D. Marcos, C. Emary, T. Brandes, and R. Aguado, New J. Phys. 12, 123009 (2010). 